\title{
Functional outcome after neonatal pyeloplasty in antenatally diagnosed uretero-pelvic junction obstruction
}

\author{
Pradnya Suhas Bendre, Parag Janardhan Karkera* ${ }^{*}$ and Monita Nanjappa
}

\begin{abstract}
Background: With routine antenatal ultrasonography, fetal hydronephrosis is commonly diagnosed. This leads to early detection of postnatal uretero-pelvic junction obstruction which may require surgical intervention. But, there is no clear consensus in the benefits of operating these patients in the neonatal age.

Methods: Aim-To study the functional outcome after pyeloplasty in neonates with antenatally diagnosed unilateral uretero-pelvic junction obstruction (UPJO). Records of all neonates $(N=48)$ who presented between 2016 and 2018 with prenatal diagnosis of unilateral UPJO and underwent a Anderson-Hyne's Pyeloplasty were retrospectively analyzed. Indications for surgery were SFU grade 3 or 4, a split renal function (SRF) $<40 \%$ on a diuretic renal scan and antero-posterior renal pelvic diameter (APD) $>2.5 \mathrm{~cm}$ with parenchymal thinning. Parenchymal thickness (PT) and APD measured by ultrasonography, and SRF measured by $99 \mathrm{~m}$ Tc-EC renal scan were compared before and after surgery.

Results: Our study comprised of 48 patients with $79.2 \%$ males $(n=38)$. UPJO affected the left side more $(n=30$, $62.5 \%$ ). The mean age at pyeloplasty was 28 days (range 26-30). The outcome was considered successful in 46 (95.84\%) patients. The APD decreased from a mean of $3.5 \mathrm{~cm}$ APD preoperatively to $1.38 \mathrm{~cm} 1$ year later which was statistically significant $(p<0.001)$. The PT increased from 3.95 to $7.1 \mathrm{~mm} 1$ year postoperatively which was significant $(p<0.001)$. The drainage pattern and SRF improved in 46 (95.84\%) patients. The SRF improved from a mean of $35.48-44.7 \% 1$ year postoperatively which was significant $(p<0.001)$.

Conclusion: Pyeloplasty done in the neonatal age for prenatally diagnosed UPJO, having SFU grade 3-4 UPJO, leads to significant improvement of SRF and PT with minimal complications. Neonatal Pyeloplasty for significant UPJO is a safe procedure which provides the kidney maximum opportunity to improve function.
\end{abstract}

Keywords: Neonatal, Pyeloplasty, Uretero-pelvic junction obstruction

\section{Background}

Uretero-pelvic junction obstruction (UPJO) is defined as an obstruction to the flow of urine from the renal pelvis to the proximal ureter. The prevalence is estimated at one per 1500 live births with a male-female ratio of 3-4 to 1 [1]. With routine antenatal ultrasonography, urological anomalies are identified as early as $12-14$ weeks

\footnotetext{
*Correspondence: drpaggy@gmail.com

Division of Pediatric Urology, Bai Jerbai Wadia Hospital for Children,

Acharya Donde Marg, Parel, Mumbai 400012, India
}

of gestation [2]. Fetal hydronephrosis, causes postnatal UPJO in $0.2-0.4 \%$ of cases [1-3] and $15-39 \%$ of them require surgical intervention [4-8]. Pyeloplasty is an effective surgical treatment to improve urinary drainage and affects postoperative renal function of the involved renal unit, but not all kidneys improve after surgical treatment $[9,10]$. At present, renal imaging modalities cannot reliably predict which renal units are at risk for permanent kidney damage, and who will improve spontaneously [11]. Some authors recommend early surgical intervention for maximal recovery of renal function 
and preservation of renal parenchyma $[8,12]$. However, some authors have shown that UPJO is relieved spontaneously in some patients kept for delayed intervention or prolonged observation and those that remain do not always lead to renal function impairment [12]. They recommend surgical option to be reserved for those with an observed decrease in renal function that can improve after pyeloplasty $[3,5]$. In this single center, retrospective, observational study, we study functional outcomes after pyeloplasty done in newborns 1 month or less in age with unilateral UPJO.

\section{Methods}

The records of all children who presented between 2016 and 2018 to our institution with prenatal diagnoses of unilateral PUJ obstruction were retrospectively analyzed. The patients $(N=48)$ included in the study were all patients who underwent a Anderson-Hyne's pyelopasty at or before 1 month of age. Indications for surgery were SFU grade 3 or 4 , a split renal function (SRF) $<40 \%$ on a diuretic renal scan with obstructed drainage, antero-posterior renal pelvic diameter (APD) $>2.5 \mathrm{~cm}$ with parenchymal thinning. Ultrasonogram (USG) was done at the end of the first week of life to confirm the diagnosis and grade according to the SFU grading of hydronephrosis and note the APD and parenchymal thickness (PT). In children with SFU grades 3 and 4, radionuclide evaluation with Technetium-99 m Ethylenedicysteine (EC) [using F-0 protocol in all patients] was done at 3 weeks of life. Obstructed drainage was considered when the EC scan showed an obstructed curve, $t 1 / 2>20 \mathrm{~min}$ and significant tracer retention after $2 \mathrm{~h}$. A voiding cystourethrogram was done only in those cases who showed ureteric or bladder abnormalities on USG. Those who did not have an obstructive pattern or had drainage at 2-h delayed film on radionuclide scan, single kidney status, bilateral disease, associated vesico-ureteric reflux, and other urological anomalies were excluded. Parents were counseled regarding the severity of hydronephrosis and the obstructive pattern.

A standard open dismembered pyeloplasty was performed by the same surgeon in all patients via the extraperitoneal approach with a mini-flank incision using 6-0 polyglactin interrupted sutures for the uretero-pelvic anastomosis. Intra-operatively, a $3 \mathrm{Fr}$ double J stent was placed in antegrade fashion across the anastomosis and a perinephric drain was kept. The stent was removed after 6-8 weeks after a follow-up USG at 1 month. USG was repeated at 3, 6, 9, 12 months post-surgery to ensure improvement noting the APD and PT.

The radionuclide scan (EC scan) was repeated at one year, at the same center using the same protocol, to assess final SRF and drainage. PT and APD measured by USG and renal function measured by $99 \mathrm{~m}$ Tc-EC renal scan were compared before and after surgery. Successful outcome was considered when all of the following were achieved, i.e., increase in PT with decrease in APD on USG with Renogram showing non-obstructive drainage pattern, reduction in $\mathrm{t} 1 / 2$ and less/no hold-up at 2 - $\mathrm{h}$ delayed films and increase of $5 \%$ in SRF from original value.

This retrospective study was approved by the institutional ethics committee (IEC-BJWHC/AP/2018/043$\mathrm{V} 1,3 / 12 / 18)$. Legal guardians of subjects signed a written, informed consent for undergoing the procedure. The study procedures complied with the guidelines provided by the Declaration of Helsinki. All original data reported in this study was entered in Microsoft Excel sheet and evaluated using the SPSS software package. The outcomes were compared using the Student $t$ test and Chisquare test.

\section{Results}

Our study comprised of 48 patients with $79.2 \%$ males $(n=38)$ and ten females. UPJO affected the left side more $(n=30,62.5 \%)$. The mean age at pyeloplasty was 28 days (range 26-30). A successful outcome was achieved in 46 (95.84\%) patients (Table 1). The mean APD before surgery was $3.5 \mathrm{~cm}$ which decreased to $1.38 \mathrm{~cm}$ after surgery which was statistically significant $(p<0.001)$. The mean PT of all renal units increased from $3.95 \mathrm{~mm}$ preoperatively to $7.1 \mathrm{~mm}$ one year postoperatively which was a significant $(p<0.001)$ increase. The APD decreased and PT increased in all cases. The SRF and drainage pattern improved in 46 (95.84\%) patients, while in two cases it stayed obstructed. $3(6.25 \%)$ patients in the study had postoperative urinary tract infection of which one $(2.08 \%)$ required re-admission and intravenous

\section{Table 1 General characteristics and complications in the study group}

\begin{tabular}{lll}
\hline & Number (N) & Percentage \% \\
\hline Sex & 38 & \\
Male & 10 & 79.2 \\
Female & & 20.8 \\
Laterality & 18 & \\
$\quad$ Right & 30 & 37.5 \\
Left & & 62.5 \\
Complications & 3 & \\
Urinary tract infections & 1 & 6.25 \\
Readmission & & 2.08 \\
Obstruction & 46 & \\
Pre-op & 2 & 95.84 \\
Post-op & & 4.16 \\
\hline
\end{tabular}


antibiotics. The unilateral SRF improved from a mean of $35.48 \%$ before surgery to $44.7 \%$ postoperatively which was significant $(p<0.001)$ (Table 2$)$. The two patients with un-improved drainage pattern and function post-surgery, had low preoperative function $(<20 \%)$. They showed anatomical improvement with decrease in APD and marginal increase in PT postoperatively (Table 3 ). They are kept on close follow-up.

\section{Discussion}

In the present study, we have tried to evaluate the benefits and drawbacks of intervening early in the neonatal age for prenatally diagnosed UPJO. To decide the optimal timing of surgery in prenatally diagnosed UPJO is difficult due to the lack of ability, to define which kidney will benefit from surgical intervention and who will have deterioration in renal function. On the one hand, the aggressive approach of operating every patient introduces risks of surgical complications to the many who would have spontaneously resolved. On the other hand, the "conservative management for all" approach puts some infants at risk for permanent loss of renal function. Several indices have been introduced to identify the need for surgical intervention. Symptoms such as pain, the APD of the affected kidney, or a decrease in SRF to below $40 \%$ of expected values and SFU grades $3-4$ are considered prime indications $[11,13,14]$. Hence, we have stuck to those indications to select patients for pyeloplasty. The role of pyeloplasty to prevent deterioration and to improve renal function is known for many years and was historically the standard of care treatment. In 1990, Ransley et al. [3] were one of the first to question the benefits of universal surgery approach. Ransley et al. reported that pyeloplasty for deterioration in function was required only in 23 out of 100 cases, while Arora et al. reported this in $23.9 \%$ cases [3, 4]. Arena et al. [5] also reported good recovery in function in those who were followed up. However, these studies also included grade 2 SFU hydronephrosis. Chertin recommended expectant management in cases of antenatally diagnosed UPJO, as it avoids unnecessary surgery and even if renal function deteriorates, delayed pyeloplasty recovers the initial functional level. However, he stressed that special efforts should be made to ensure that patients are not lost to follow-up [15]. Most authors acknowledge the importance of close surveillance, with a maximum interval between diagnostic tests of 3 months for the first 2 years of life, so that any obstruction can be relieved promptly and kidney damage avoided [15]. In developing countries like India, with a sizeable rural and illiterate population with poor compliance, close follow-up may not be feasible.

Although there are several publications supporting initial conservative treatment of UPJO diagnosed prenatally, several authors have expressed concern about loss of renal function during expectant management. In 1998, Conford and Rickwood and Khemchandani in 2019 felt that in patients born with UPJO and impaired renal function, pyeloplasty failed to significantly improve function, possibly because of cortical loss. They felt there is a small but significant risk of a modest loss of renal function in them [9, 16]. Arena et al. [5] concluded that high-grade hydronephrosis due to unilateral UPJO is unlikely to resolve spontaneously and is often accompanied by a loss of renal function during the first years of life. McAleer

Table 2 Comparison of antero-posterior diameter, parenchymal thickness and split renal function before and after pyeloplasty

\begin{tabular}{|c|c|c|c|c|c|c|}
\hline \multirow[t]{2}{*}{ Parameter } & \multicolumn{2}{|c|}{ Antero-posterior diameter $(\mathrm{cm})$} & \multicolumn{2}{|c|}{ Parenchymal thickness (mm) } & \multicolumn{2}{|c|}{ Split renal function (\%) } \\
\hline & Pre-oP & Post-op & Pre-op & Post-op & Pre-op & Post-op \\
\hline \multicolumn{7}{|l|}{ Number $(N=48)$} \\
\hline Mean & $3.50 \pm 1.392$ & $1.38 \pm 1.31$ & $3.95 \pm 1.213$ & $7.14 \pm 1.889$ & $35.48 \pm 9.501$ & $44.7 \pm 8.73$ \\
\hline Mean difference & 2.121 & & 3.191 & & 9.217 & \\
\hline Standard error of difference & 0.386 & & 0.378 & & 1.886 & \\
\hline$p$ value & $<0.001$ & & $<0.001$ & & $<0.001$ & \\
\hline
\end{tabular}

Table 3 Details of patients with persistent obstructed curve post-pyeloplasty

\begin{tabular}{|c|c|c|c|c|c|c|}
\hline \multirow[t]{2}{*}{ Patient } & \multicolumn{2}{|c|}{ Antero-posterior diameter $(\mathrm{cm})$} & \multicolumn{2}{|c|}{ Parenchymal thickness (mm) } & \multicolumn{2}{|c|}{ Split renal function (\%) } \\
\hline & Pre-op & Post-op & Pre-op & Post-op & Pre-op & Post-op \\
\hline A & 2.5 & 0.8 & 3 & 8 & 20 & 19.5 \\
\hline B & 2.9 & 1.2 & 4 & 7 & 18 & 17.3 \\
\hline
\end{tabular}


and Kaplan noted that a third of patients with prenatal UPJO on conservative management ultimately required pyeloplasty. They found no recovery in renal function after surgery and suggested a more aggressive approach and emphasized that loss of function could be avoided by early surgery [7]. In another study, Kim and colleagues compared renal function before and after pyeloplasty in children with unilateral UPJO according to the children's age. They found surgical intervention before 1 year of age was a significant factor for recovery of PT [17]. Babu et al. have shown that while renal function improves significantly in those undergoing early pyeloplasty, delayed surgery leads to marginal but significant loss of function. They advised an early surgery toward those presenting with high-grade hydronephrosis [18]. Chandrasekharam et al. [19] reported that infants $<1$ year of age showed significant improvement in renal function after pyeloplasty compared with older children. Roth and Gonzales [20] recommended early pyeloplasty with the belief that rapidly maturing infant kidney has the greatest potential to improve. Palmer et al. performed a multicenter prospective randomized study showing that patients who underwent surgical correction had significantly improved renal drainage compared with those who continued on an observation protocol. They insisted that early surgical correction improves renal drainage, which is a clear benefit of early surgical repair [8]. Our study findings concurs with the findings of these authors [7-9, 16-19], as it reveals significant improvement in renal function, decrease in hydronephrosis and increase in parenchymal thickness when early aggressive surgical approach is implemented.

Some authors have co-related increase in re-admission rates with early surgery, but we have had minimal readmission rates, minimal complication with good results [21]. Ceyhan et al. in his study stressed that transanastomotic drainage decreased complications, while preoperative diversions increased complications and chances of recurrence. In all our surgeries too, we went in for upfront pyeloplasty with a DJ stent without preoperative diversion [22].

Although, no consensus exists about the preoperative factors that predict recovery of renal function in children with UPJO, our results show that early age at the time of surgery is related to the recovery of PT and SRF in these children. However, this short-term study had some limitations viz. small sample size, non-randomized design and no placebo control group. The exact effect of patient age at the time of surgery should be verified with a prospective, randomized, long-term follow-up study with a large number of cases. Although, the number of patients included in the study was small, this may be largest study including only neonates undergoing pyeloplasty.

\section{Conclusion}

Early pyeloplasty in the neonatal age done in prenatally diagnosed SFU grade 3-4 UPJO leads to significant improvement of SRF, parenchymal thickness with minimal complications. Hence, we recommend early neonatal pyeloplasty as a safe option in those with SFU grade 3-4 UPJO and obstructive renogram on postnatal evaluation. This gives the growing kidney the best opportunity to recover its function.

\section{Abbreviations}

UPJO: Uretero-pelvic junction obstruction; SFU: Society of Fetal Urology; SRF: Split renal function; APD: Antero-posterior renal pelvic diameter; USG: Ultrasonogram; PT: Parenchymal thickness.

\section{Acknowledgements}

Not applicable.

\section{Authors' contributions}

PB involved in concept, design of study and editing of manuscript. PK involved in analysis of data, drafting and editing the manuscript. MN involved in collection of data, drafting the manuscript. All authors have read and approved the final manuscript.

\section{Funding}

Not applicable.

Availability of data and materials

Yes, all data generated or analysed during this study are included in this published article.

\section{Ethics approval and consent to participate}

Ethics approval taken from Institutional Ethics Committee—Bai Jerbai Wadia Hospital for Children Registration Number-(IEC-BJWHC/AP/2018/043$\mathrm{V} 1,3 / 12 / 18)$. Yes, in written format from parents/legal guardian as all patients were $<1$ month of age.

\section{Consent for publication}

Not applicable.

Competing interests

The authors declare that they have no competing interests.

Received: 7 Auqust 2020 Accepted: 2 January 2021

Published online: 21 January 2021

References

1. Schlomer BJ, Cohen RA, Baskin LS (2014) Renal imaging: congenital anomalies of the kidney and urinary tract. In: Palmer L, Palmer J (eds) Pediatric and adolescent urologic imaging. Springer, New York, NY, pp 155-198. https://doi.org/10.1007/978-1-4614-8654-1_9

2. Dias T, Sairam S, Kumarasiri S (2014) Ultrasound diagnosis of fetal renal abnormalities. Best Pract Res Clin Obstet Gynaecol 28(3):403-415. https:// doi.org/10.1016/j.bpobgyn.2014.01.009 (Epub 2014 Jan 29)

3. Ransley P, Dhillon H, Gordon I et al (1990) The postnatal management of hydronephrosis diagnosed by prenatal ultrasound. J Urol 144:584-587

4. Arora S, Yadav P, Kumar M, Singh SK, Sureka SK, Mittal V et al (2015) Predictors for the need of surgery in antenatally detected hydronephrosis due to UPJ obstruction-a prospective multivariate analysis. J Pediatr Urol 11(5):248.e1-248.e5. https://doi.org/10.1016/j.jpurol.2015.02.008 (Epub 2015 Mar 13)

5. Arena S, Chimenz R, Antonelli E, Peri FM, Romeo P, Impellizzeri P et al (2018) A long-term follow-up in conservative management of unilateral 
ureteropelvic junction obstruction with poor drainage and good renal function. Eur J Pediatr. https://doi.org/10.1007/s00431-018-3239-2

6. Lee JN, Kang JK, Jeong SY, Lee SM, Cho MH, Ha Y-S et al (2018) Predictive value of cortical transit time on MAG3 for surgery in antenatally detected unilateral hydronephrosis caused by ureteropelvic junction stenosis. J Pediatr Urol 14(1):55.e1-55.e6. https://doi.org/10.1016/j.jpuro 1.2017.08.009

7. McAleer IM, Kaplan GW (1999) Renal function before and after pyeloplasty: Does it improve? J Urol 162:1041-1044

8. Palmer LS, Maizels M, Cartwright PC et al (1998) Surgery versus observation for managing obstructive grade 3-4 unilateral hydronephrosis: a report from the Society for Fetal Urology. J Urol 159:222-225

9. Cornford PA, Rickwood AMK (1998) Functional results of pyeloplasty in patients with antenatally diagnosed pelviureteric junction obstruction. BJU Int 81:152-155

10. Salih EM (2015) Morphological and functional outcome of dismembered pyeloplasty in children with unilateral ureteropelvic junction obstruction. Afr J Urol 21:174-180. https://doi.org/10.1016/j.afju.2015.04.003

11. Chiodini B, Ghassemi M, Khelif K et al (2019) Clinical outcome of children with antenatally diagnosed hydronephrosis. Front Pediatr 7:103

12. Tabari AK, Atqiaee K, Mohajerzadeh L et al (2019) Early pyeloplasty versus conservative management of severe ureteropelvic junction obstruction in asymptomatic infants. J Pediatr Surg 55:1936-1940. https://doi. org/10.1016/j.jpedsurg.2019.08.006

13. Chertin B, Pollack A, Koulikov D, Rabinowitz R, Hain D, Hadas-Halpren I et al (2006) Conservative treatment of ureteropelvic junction obstruction in children with antenatal diagnosis of hydronephrosis: lessons learned after 16 years of followup. Eur Urol 49:734-739

14. Cheng H-L (2014) Surgical indications for unilateral neonatal hydronephrosis in considering ureteropelvic junction obstruction. Urol Sci 25:73-76
15. Chertin B, Rolle U, Farkas A, Puri P (2002) Does delaying pyeloplasty affect renal function in children with a prenatal diagnosis of pelvi-ureteric junction obstruction? BJU Int 90:72-75

16. Khemchandani SI (2019) Outcome analysis of pediatric pyeloplasty in varied presentation in developing countries. Urol Sci 30:276-280

17. Kim SO, Yu HS, Hwang IS, Hwang EC, Kang TW, Kwon D (2014) Early pyeloplasty for recovery of parenchymal thickness in children with unilateral ureteropelvic junction obstruction. Urol Int 92(4):473-476

18. Babu R, Rathish VR, Sai V (2015) Functional outcomes of early versus delayed pyeloplasty in prenatally diagnosed pelvi-ureteric junction obstruction. J Pediatr Urol. 11(2):63 e1-5

19. Chandrasekharam VV, Srinivas M, Bal CS et al (2001) Functional outcome after pyeloplasty for unilateral symptomatic hydronephrosis. Pediatr Surg Int 17:524-527

20. Roth DR, Gonzales ET Jr (1983) Management of ureteropelvic junction obstruction in infants. J Urol 129:108-110

21. Vemulakonda VM, Wilcox DT, Combleholme TM, Bronsert M (2015) Factors associated with age at pyeloplasty in children with ureteropelvic junction obstruction. Pediatr Surg Int. https://doi.org/10.1007/s0038 3-015-3748-2

22. Ceyhan E, lleri F, Ceylan T, Aydin AM, Dogan HM, Tekgul S (2019) Predictors of recurrence and complications in pediatric pyeloplasty. Urology 126:187191. https://doi.org/10.1016/j.urology.2019.01.014

\section{Publisher's Note}

Springer Nature remains neutral with regard to jurisdictional claims in published maps and institutional affiliations.

\section{Submit your manuscript to a SpringerOpen ${ }^{\circ}$ journal and benefit from:}

- Convenient online submission

- Rigorous peer review

- Open access: articles freely available online

- High visibility within the field

- Retaining the copyright to your article

Submit your next manuscript at $\boldsymbol{\nabla}$ springeropen.com 